\title{
Pituitary Tumor Associated with Situs Inversus of the Optic Nerve Head
}

\author{
T. David Williams, \\ OD, MS, PhD \\ Professor Emeritus \\ School of Optometry \\ University of Waterloo
}

\begin{abstract}
This report present further developments in a patient with an unusual vascular pattern on both optic nerve heads: the trunks of the central retinal vessels appear on the temporal side of the nerve head (situs inversus of the blood vessels of the nerve head). This vascular pattern was also present (to only slightly varying degrees) in all six of his children (two girls and four boys). Twenty-one years after the first presentation of this patient, he developed a non-secretory pituitary tumor. This case suggests that congenital nerve head anomalies may be markers for potential intracranial tumors later in life.
\end{abstract}

\section{INTRODUCTION}

Embryonic development of the pituitary gland and optic nerve head proceed within the same time-frame as development of the central nervous system (approximately 40 days after conception). The optic vesicle and stalk are outgrowths of the diencephalon, as is the neural portion of the pituitary gland (neurohypophysis). The anterior portion of the pituitary gland (adenohypophysis) and the anterior segment of the eye are derived from the surface ectoderm and neural crest. Thus, disturbances in growth occurring at around the 40-day stage may affect the development of both the optic nerve and the pituitary gland.

In an ophthalmic context, the term situs inversus has been used to describe tilted nerve heads, a temporal appearance of the central retinal vessel trunks, or both. This condition can also be described in terms of the presence or absence of a crescent of visible sclera, usually on the side opposite the most elevated portion of the nerve head. This group of findings raises questions about the processes that are underway while these structures are being formed. Apple and co-workers ${ }^{1}$ described situs inversus of the retinal vessels as an element in the tilted disk syndrome; however, in the present cases, there were neither observable tilts to the nerve heads nor crescents of hypopigmentation. Thus, situsinversus of the retinalvessels mayoccurindependently of the usually inferiorly located colobomas described by Apple, and may not be caused by disturbances in the closure of the fetal fissure. Situs inversus of the central retinal vessels is also seen in Williams (elfin-face) syndrome ${ }^{2}$ and Ehlers-Danlos syndrome. ${ }^{3}$

In some cases, tilted nerve heads by themselves are associated with visual field loss, and this is often of a bitemporal nature, ${ }^{4}$ without respect for the vertical midline. Considering that the axons from the nasal retina develop long before those from the temporal retina, the presence of bitemporal field losses argues for a disturbance $>>>$ that occurs $<<<$ comparatively early in the embryonic development of the visual system. 
Fig. 1. Fundus photos of the patient's family. Top (inside the box): photos on the left show the patient; photos on the right show his wife. Following pairs are in birth order, starting at the second row - On the left: two daughters, one son. On the right: three sons. $R$ : right eyes; L: left eyes.

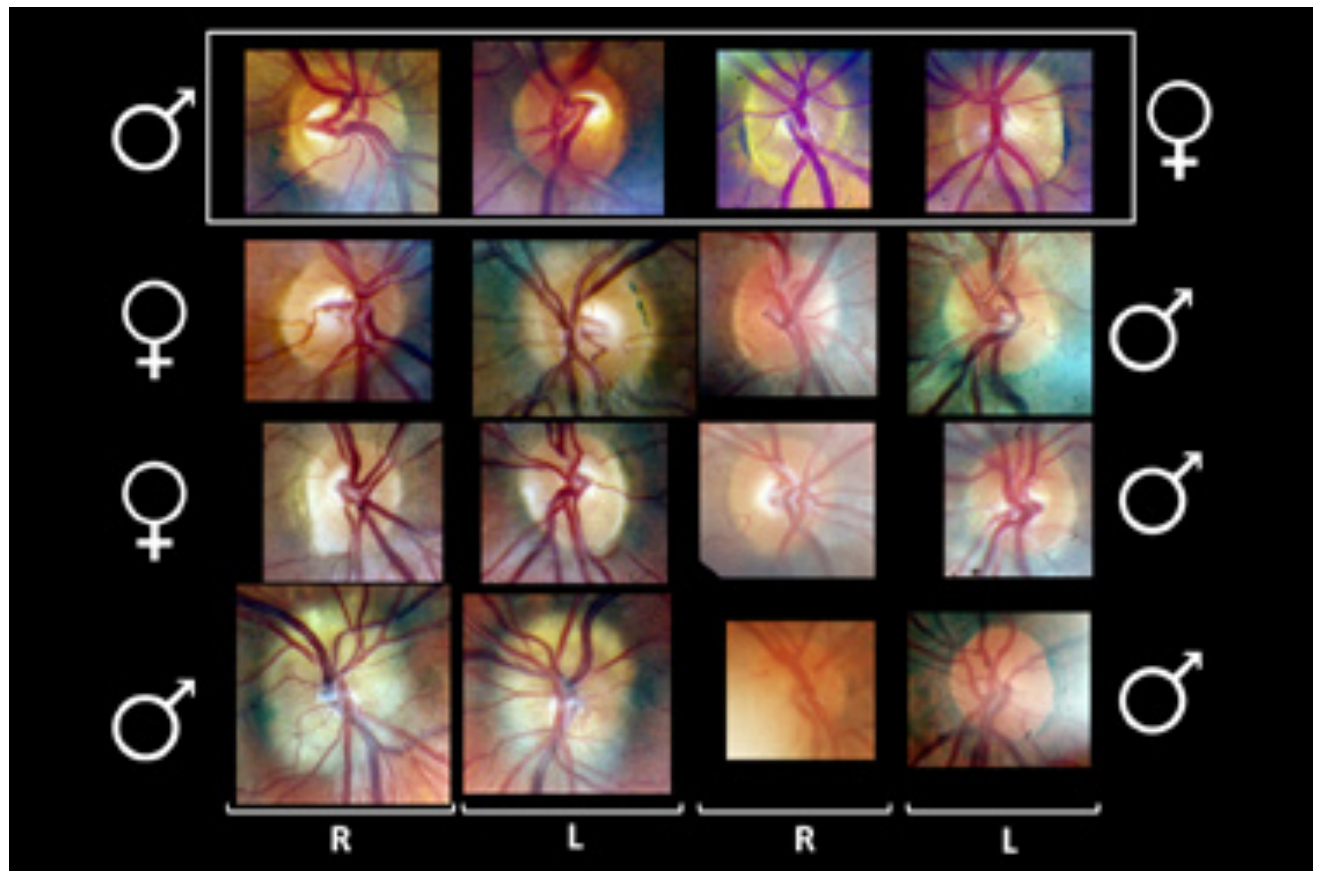

Fig. 2: Octopus visual field test results for the left eye, 15 days prior to surgery for macroadenoma.

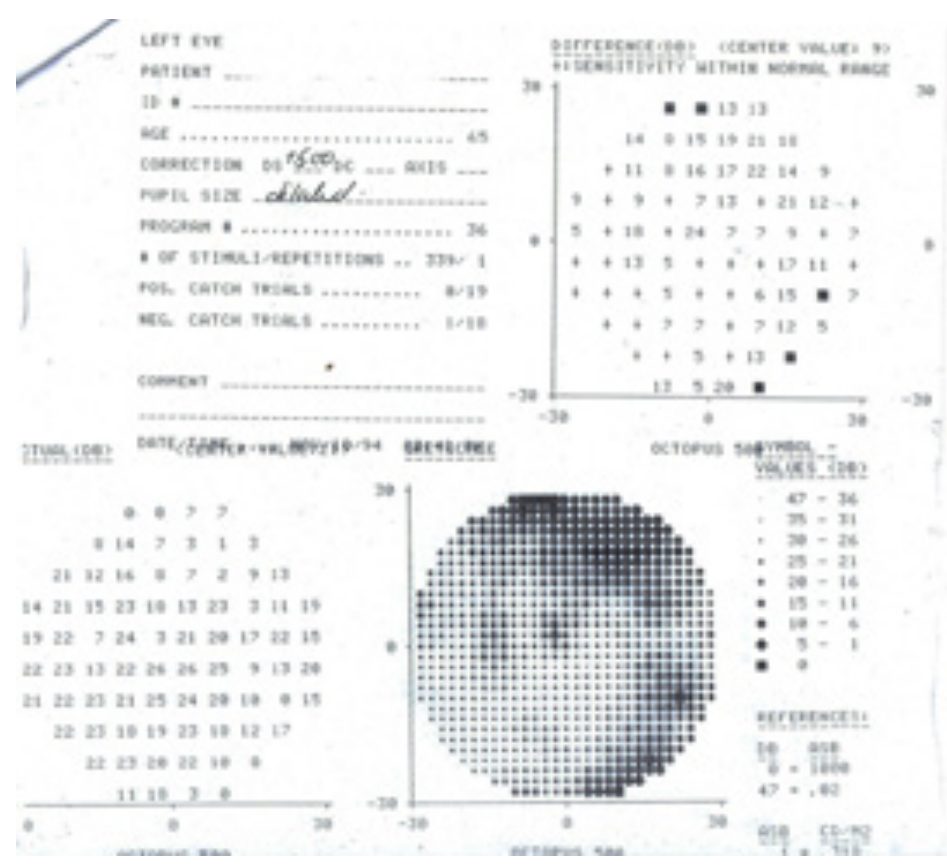




\section{CASE REPORT}

In 1973, a 43-year-old male showed an unusual entrance of the central retinal vessels on his nerve heads (Fig. 1 top left). Corrected visual acuity for the left eye at that time was 20/15, while that for the right eye was on the order of 20/200. The poor acuity in the right eye may have been related to a car accident at age 4 . The right eye also showed a unilateral esotropia. Both eyes were hyperopic in the $6 \mathrm{D}$ range.

Since the rest of his family were being seen at the University of Waterloo Optometry Clinic at that time, their nerve heads were also photographed. His wife is shown in Fig. 1 top right. The couple had two daughters and four sons. All showed some degree of situs inversus of the nerve head blood vessels (Fig. 1 balance of photos). This is consistent with an autosomal dominant pattern of inheritance. These findings have been reported previously. ${ }^{5}$

In 1994, at age $64 \mathrm{y}$, the patient had a non-secretory (i.e. null cell) pituitary macroadenoma removed via transsphenoidal resection. The sequence of events leading to discovery of the tumor was as follows. The patient underwent cataract surgery in September 1994. Over the two months following cataract surgery, he experienced a decrease in left visual acuity from 20/50 to 20/200. Cystoid macular edema was ruled out. Visual field testing for the left eye (Fig. 2) showed decreased sensitivity in the superior nasal field, close to the center of the field. There was no afferent pupil defect, and extraocular muscle function was normal. When the patient mentioned to his eye surgeon that his brother had been diagnosed with a brain tumor in 1990, the eye surgeon ordered a brain scan to rule out a central cause for the loss of acuity. This scan revealed a macroadenoma, which was removed in late November 1994. Further visual field testing prior to and following removal of the macroadenoma (Fig. 3) showed a greatly improved left visual field after surgery; left visual acuity improved to $20 / 25$, where it remains to date.

The vascular pattern of the optic nerve head is established by the seventh to eighth month of gestation. ${ }^{6}$ Although the nerve head anomaly in this patient appears to have been present at birth, the pituitary problem was not noted until 64 years later.

Fig. 3. Goldmann visual field test results closer to the time of surgery: $A$ and B show the right and left field results 3 days prior to surgery; $C$ is the left field result 3 days after surgery. In $A$, there is a central defect extending superotemporally. In $B$, there is a left superotemporal field defect. In $C$, the superotemporal field defect has resolved. Shaded areas are scotomas. Inner solid lines show isopters obtained with the Goldmann II-4 target; outer, broken lines are norms for 65 year olds obtained with the Goldmann III-3 target, which has a stimulus value equivalent to the II-4 target.
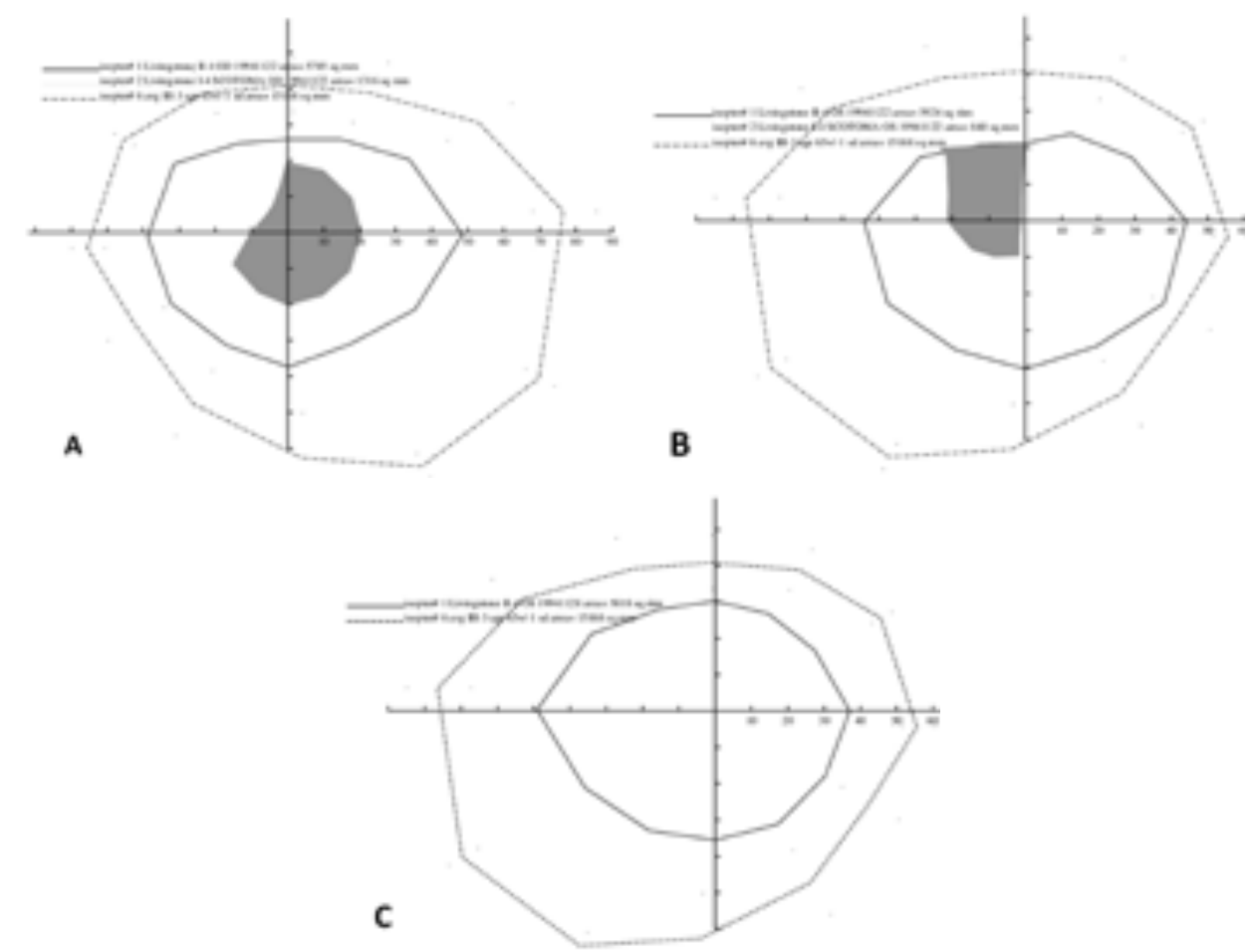
Fig. 4: Hypothetical horizontal cross-section through the right nerve head of the patient. The strong temporal direction of the optic nerve causes the centrally-located central retinal vein and artery to appear toward the temporal edge of the nerve head. The dashed pair of lines in the lower part of the diagram represent the lamina cribrosa, while the curved dashed line in the upper part of the diagram represents the nasal part of the cupping. (Reproduced, with permission, from Williams TD. Congenital malformations of the optic nerve head. Am J Optom Physiol Opt 1978;55(10):706-18.)

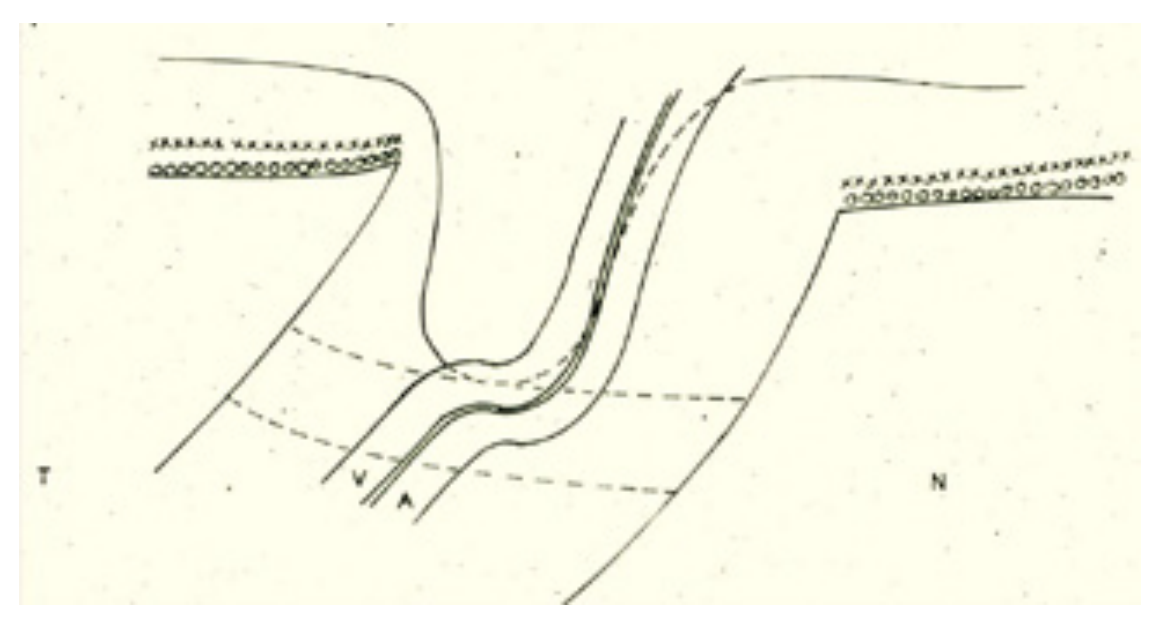

\section{DISCUSSION}

The association of nerve head colobomas and prolactinoma has been previously reported.7 Colobomas may be associated with a wide range of congenital abnormalities, including cardiovascular, gastrointestinal, skeletal, and urogenital abnormalities. Hypoplasia or aplasia of the trigeminal nerve may occur in patients with nerve head colobomas. ${ }^{8}$ I have previously reported another patient with situs inversus and chromophobe adenoma. ${ }^{9}$

Optic nerve anomalies can be associated with anomalies in the development of the central nervous system. An example is septo-optic dysplasia, ${ }^{10}$ which features various combinations of optic nerve hypoplasia, pituitary hormone deficiencies, and midline abnormalities such as absence of the septum pellucidum. Optic nerve hypoplasia may also be associated with posterior pituitary ectopia and thinning of the corpus callosum. ${ }^{11}$ For some patients with optic nerve anomalies, the associated CNS anomalies are present at birth, as in the case of the basal encephalocele associated with morning glory nerve head. ${ }^{12}$ For other patients, CNS anomalies such as craniopharyngioma may not develop until adulthood. The embryonic basis for craniopharyngioma is clear. Craniopharyngioma, which arises from the adenohypophyseal placode (i.e. Rathke's pouch), may be associated with various congenital nerve head abnormalities (optic nerve hypoplasia, tilted disc). ${ }^{13}$ One theory suggests that when craniopharyngioma presents in adulthood, this signifies that some embryonic tissue from the pharynx remained dormant (embryonic rest) for years, and then resumed its growth. ${ }^{14}$ The posterior pituitary (neurohypophysis) can be abnormal, even ectopic, in some people with morning glory nerve head..$^{15}$ Morning glory nerve head is also associated with Down syndrome, ${ }^{16}$ hypertelorism, cleft lip and palate, basal encephalocele, agenesis of the corpus callosum, defects in the floor of the sella turcica, renal anomalies and the CHARGE syndrome (Coloboma, Heart defects, Atresia of the nasal choanae, Retardation of growth and/or development, Genitourinary abnormalities, Ear deformities). ${ }^{17}$

Situs inversus of the retinal vessels of the optic nerve head is also likely associated with a strong temporal direction of the optic nerve as it leaves the globe. ${ }^{18}$ Figure 4 shows a hypothetical horizontal cross-section of the present patient's right optic nerve head. Upon ophthalmoscopic examination, the trunks of the central retinal vessels will first be seen within the cupping close to the temporal edge of the nerve head. Under normal circumstances, one would expect the trunks of the central retinal vessels to be either at the center of the nerve head (where they would not be seen at all) or, as with many myopic fundi, the trunks of the vessels appear to have been tucked away on the nasal side of the nerve head, owing to a somewhat nasal direction of the optic nerve. This discrepancy led to the term 'situs inversus of the central retinal vessels', since the patient's right eye had a vascular pattern that was more like that expected in a left eye. 
It may be that the optic nerve is pushed in this temporal direction by a disproportion in either the number or rate of growth of the ganglion cell axons, with fewer axons present on the temporal side of the nerve head and more axons present on the nasal side of the developing nerve. This mechanism may explain the great variation in the apparent direction of optic nerves: all directions appear to be possible.

The normal optic nerve, the temporally-directed optic nerve, and the hypoplastic optic nerve may lie along a spectrum of ganglion cell axon development: if there is no interference, the optic nerve will be normal, oriented at right angles to the globe of the definitive eyeball; if there is more interference with the growth of axons from the temporal retina than in other areas, then the partially hypoplastic optic nerve will be oriented temporally as it leaves the globe; if there is overall interference with axon growth, there will be an overall hypoplastic optic nerve, also oriented at right angles to the globe. In the partially hypoplastic case, the trunks of the central retinal vessels will appear toward the temporal edge of the disc.

The late appearance of an anterior pituitary tumor in the present case suggests the possibility of a similar embryonic rest in the region of the pituitary gland, and that the growth disturbance which caused placement of this anomalous tissue may have also caused the congenital anomaly involving the optic nerve.

There is no basis for concluding that all patients with situs inversus of the retinal vessels will later develop a pituitary tumor. This vascular pattern is relatively uncommon (occurring in $5 \%$ of eyes without a tilted disc ${ }^{19}$ ), while pituitary tumors are found one in six autopsy cases. ${ }^{20}$ Nevertheless, patients who present with nerve head anomalies require further investigation to rule out other intracranial or systemic abnormalities.

\section{ACKNOWLEDGEMENT}

I thank Dr. Hugh G. Jellie (Kitchener) for his notes leading to the discovery of the patient's pituitary tumor. Goldmann fields in Fig. 3 are drawn from notes by Dr. David Nicolle, London Health Sciences Centre.

\section{REFERENCES}

1. Apple DJ, Rabb MF, Walsh PM. Congenital anomalies of the optic disc. Surv Ophthalmol 1982;27(1):3-41.

2. Greenberg F, Lewis RA. The Williams Syndrome, spectrum and significance of ocular features. Ophthalmology 1998;95:1608-12.

3. Forman AR. Situs inversus of the optic disc in Ehlers-Danlos Syndrome, type III. Ophthalmology 1979;86:844-6

4. Witmer MT, Margo CE, Drucker M. Tilted optic disks. Surv Ophthalmol 2010;55(5):403-8.

5. Williams TD. Congenital malformations of the optic nerve head. Am J Optom Physiol Opt 1978;55(10):706-18.

6. Taylor D. Congenital tumours of the anterior visual system with dysplasia of the optic discs. Br J Ophthalmol 1982;66:455-63

7. Williams TD. Systemic abnormalities associated with iris/nerve head/choroidal/retinal coloboma. Optom Vis Sci 1996;72(7):506-10.

8. Villanueva O, Atkinson DS, Lambert SR. Trigeminal nerve hypoplasia and aplasia in children with goldenhar syndrome and corneal hypoesthesia. J AAPOS 2005;9 (2):202-4.

9. Williams TD. Nerve head anomaly associated with pituitary tumor. Can J Optom 1987;49(1):18-9.

10. Koizumi M, Ida S, Shoji Y, Etani Y, Hatsukawa Y, Okamoto N. Endocrine status of patients with septo-optic dysplasia: fourteen Japanese cases. Clin Pediatr Endocrinol 2017; 26(2): 89-98.

11. Dutton GN. Congenital disorders of the optic nerve: excavations and hypoplasia. Eye 2004;18:1038-1048.
12. Sasani M, Ozer AF, Aydin AL. Endoscopic treatment of trans-sellar trans-sphenoidal encephalocele associated with morning glory syndrome presenting with non-traumatic cerebrospinal fluid rhinorrhea. J Neurosurg Sci 2009;53(1):31-5.

13. Taylor D. Congenital tumours of the anterior visual system with dysplasia of the optic discs. Br J Ophthalmol 1982;66(7):455-63.

14. Muller HL. Craniopharyngioma. Endocr Rev 2014;35:513-43.

15. Pierre-Filho PP, Limeira-Soares PH, Marcondes AM. Morning glory syndrome associated with posterior pituitary ectopia and hypopituitarism. Acta Ophthalmol Scand. 2004;82:89-92.

16. Altun A, Altun G, Kurna SA, Olcaysu OO, Aki SF. Unilateral morning glory optic disc anomaly in a case with Down syndrome. BMC Ophthalmol 2014;14:48.

17. Eustis HS, Sanders MR, Zimmerman T. Morning glory syndrome in children. Association with endocrine and central nervous system anomalies. Arch Ophthalmol 1994;112:204-7.

18. Williams TD. Speculations on the direction of the optic nerve, Am J Optom Physiol Opt 1983;60(4):303-7.

19. Vongphanit J, Mitchell P, Wang JJ. Population prevalence of tilted optic disks and the relationship of this sign to refractive error. Am J Ophthalmol 2002;133(5):679-85.

20. Ezzat S, Asa SL, Couldwell WT, et al. The prevalence of pituitary adenomas. A systematic review. Cancer 2004;101(3):613-9. 